\title{
Auswirkungen der COVID-19 Pandemie auf die Arbeit mit Moodle - Aktuelles aus Österreichs Schulen
}

\author{
"robert.schrenk@lernmanagement.at, Zentrum für Lernmanagement, Pädagogische Hochschule OÖ
}

eingereicht am: 19.03.2021, akzeptiert am: 18.04.2021

\begin{abstract}
Ausgelöst durch die COVID-19 Pandemie und damit einhergehende Schulschließungen waren viele Pädagog*innen gezwungen auf digitale Lehre umzustellen. Unter Begriffen wie „Home Schooling“ oder „Distance Learning“ firmierte in diesen Zeiten der Unterricht. Auch in Österreich wurden Schulen aufgrund der Pandemie mehrfach geschlossen. Dieser massive Einschnitt in den Berufsalltag von Lehrer*innen hat naturgemäß Auswirkungen auf die Art des Lehrens und Lernens sowie auf die Wahl der genutzten digitalen Plattformen und Werkzeuge.

In diesem Artikel wird auf Basis vorhandenen Zahlenmaterials der Moodle-Lernplattform eduvidual.at in Anlehnung an das SAMR-Modell eine Analyse vorgenommen, wie Lehrer*innen während des Distance Learnings die Lernplattform verwendet haben, und ob damit zu rechnen ist, dass sich pädagogische Herangehensweisen geändert haben.
\end{abstract}

Keywords: Distance Learning, Lernplattform, Moodle, eEducation, SAMR-model

\section{Impact of the COVID-19 pandemic on the work with Moodle - News from Austrian schools}

Triggered by the COVID-19 pandemic and the resulting school closures, many educators were forced to switch to digital teaching. During these times, teaching was referred to as „home schooling“ or „distance learning“. In Austria, schools were closed several times due to the pandemic, too. This massive impact on the daily work of teachers has affected the way of teaching and learning as well as the choice of digital platforms and tools used.

Based on available figures from the Moodle learning platform eduvidual.at and following the SAMR model, an analysis is made of how teachers used the learning platform during distance learning, and whether it can be expected that pedagogical approaches have changed.

Keywords: distance learning, learning platform, Moodle, eEducation, SAMR-model

\section{Einleitung}

Nach einer Studie aus Norwegen, hätte es dort aufgrund von Schulschließungen „mehr Kreativität beim Lernen, bessere Fortschritte, mehr nützliches Feedback und mehr Unabhängigkeit beim Lernen“ gegeben (Bubb \& Jones 2020). Insbesondere die Schaffung „kreativer Aufgaben“ wurde von Schüler*innen und Erziehungsberechtigten gelobt. Diese „kreativen Aufgaben" waren aber nicht die Folge von Schulschließungen, sondern die untersuchten Schulen hatten bereits zuvor an einer Transformation der eigenen Pädagogik gearbeitet. Man war sogar besorgt, dass die Schulschließungen die bisher erreichten Fortschritte zunichtemachen könnten. Im Gegenteil hat der Einsatz von Technologie im Distance Learning die
Lehrer*innen dazu motiviert, noch mehr auf kreative Aufgaben zu setzen. Der Erfolg dieser norwegischen Schulen erklärt sich also nicht aus der Tatsache, dass überhaupt digitale Technologien eingesetzt wurden, sondern daraus, dass eine veränderte Pädagogik durch die Technologie einen weiteren Förderungsimpuls bekommen hat.

In Österreich war die Sorge vor negativen Auswirkungen durch Schulschließungen ebenfalls groß. So wäre zu befürchteten, dass die Bildungsschere zwischen Arm und Reich weiter aufgehen würde (Madner 2020), Schüler*innen vielerorts keine eigenen digitalen Geräte oder Internetanschluss zur Verfügung hätten, aber auch, dass schlicht die digitalen Kompetenzen fehlen würden, damit der Unterricht ohne qualitative Einbußen digital stattfinden könne (Ottacher et al. 2020). 
Gerade der Erwerb der digitalen Kompetenzen bei Lehrer*innen war über viele Jahre hinweg auf Freiwilligkeit aufgebaut. Die Nutzung digitaler Technologien für den Unterricht wurde an Schulen in Österreich von einer verhältnismäßig kleinen Gruppe an Personen vorangetrieben. Während das Thema seit der Gründung des National Competence Center e-Education Austria im Jahr 2016 zunehmende Bedeutung erfahren hat, war die Beschäftigung mit digitalen Werkzeugen aber dennoch freiwillig.

Lehrer*innen in Österreich, die schon vor der COVID-19 Pandemie mit Moodle gearbeitet haben, hatten eine intrinsische Motivation sich die digitalen Technologien nutzbar zu machen und qualitative Verbesserungen ihres Unterrichts zu erreichen. Viele haben daher ebenfalls innovative Aufgabenformate erarbeitet, die das Lernen bspw. mit kooperativen Aufgaben, Game Based Learning oder Gamification interessanter machten. Es gab also bereits eine Mischung aus herkömmlichen und neuartigen Aufgaben, die in Moodle zumeist mit entsprechend spezialisierten Modulen umgesetzt wurden.

Ausgelöst durch COVID-19 wurden die Schulen in Österreich dann drei Mal für jeweils 1-2 Monate geschlossen, wodurch auf einen Schlag alle Lehrer*innen gezwungen waren, auf digitale Lehre umzustellen. Konkret war der erste Lockdown zwischen März und April 2020, der zweite Lockdown im November 2020 und der dritte Lockdown im Jänner 2021. Viele Lehrer*innen haben erst zu diesem Zeitpunkt mit digitaler Lehre begonnen.

$\mathrm{Zu}$ Beginn der Pandemie identifizierten Jekel, Oberrauch und Breitfuss-Horner (2020: 60) folgendes Problemfeld beim Distance Learning: „Sowohl den Schülerinnen und Schülern als auch den Lehrpersonen müssen Arbeitsmethoden beigebracht werden, die über die Konsumption von Online-Videos (oder anderen Informationsressourcen) und der Reproduktion/Reorganisation zentraler inhaltlicher Aussagen hinausgehen."

\section{SAMR-Modell und Moodle-Aktivitäten}

Das SAMR-Modell von Puentedura (2006) kann als Instrument zur Reflexion dessen herangezogen werden, welchen Mehrwert der Einsatz digitaler Technologien für das Lernen ermöglicht. Dieses Modell besagt, dass digitale Technologien zumindest (1) als Ersetzung von Analogem herhalten (Substitution), (2) eine Verbesserung ermöglichen (Augmentation), (3) durch ihren Einsatz die Lernaufgaben transformiert werden (Modification), oder (4) komplett neue Aufgaben definiert werden können, die ohne Technologie nicht möglich wären (Redefinition).

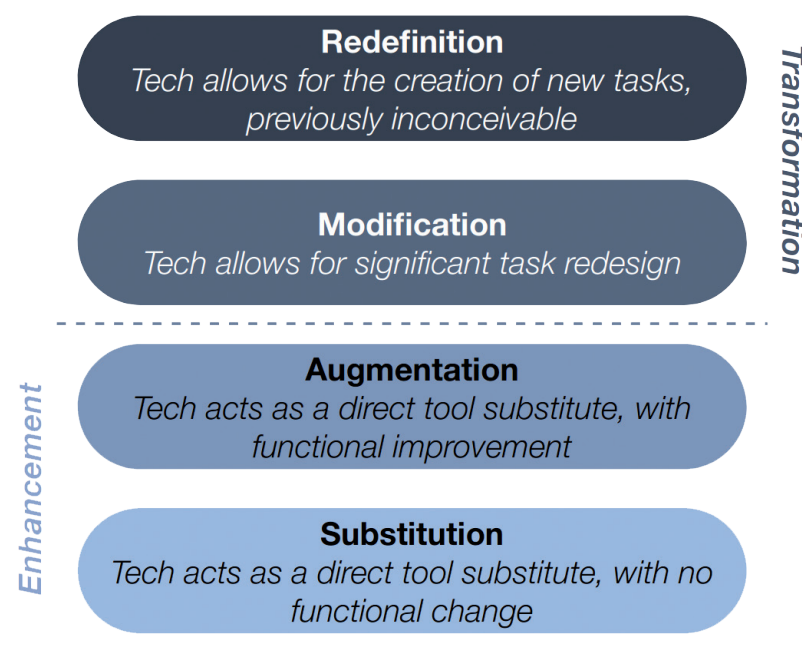

Abb. 1: SAMR-Modell (Puentedura 2013)

Auf der Ebene der Ersetzung (Substitution) würden beispielsweise Arbeitsblätter statt in Form einer Papierkopie als Datei verschickt und als ebensolche wieder eingesammelt werden. Der Präsenzunterricht würde durch Videokonferenzen ersetzt werden. So wurde schon in der Vergangenheit der "falsche“ Einsatz von Moodle als „PDF-Schleuder“ kritisiert (Jäger et al. 2014: 494), also das hauptsächliche Arbeiten mit zum Download angebotenen Dateien. Obwohl sich diese Bezeichnung darauf bezieht, dass Lehrpersonen Moodle-Kurse nur zur Verteilung ihrer Materialien nutzen, zeigte die Erfahrung aus den Schulschließungen, dass Schüler*innen im Home Schooling in einem großen Ausmaß Arbeitsblätter analog ausgefüllt und als Foto in herkömmlichen Moodle-Aufgaben hochladen sollten. In diesem Sinne funktioniert die „PDFSchleuder" neuerdings auch in die andere Richtung. Videokonferenzen spielten vor den Schulschließungen eine sehr untergeordnete Rolle und gewannen erst jetzt an Bedeutung. Sie sind ein Ersatz für Präsenzunterricht und es ist fraglich, ob ihre Bedeutung bleibt, wenn die Schulen wieder offen sind.

Verbesserung (Augmentation) lässt sich in Form digitaler Aktivitäten erreichen, beispielsweise mittels Moodle-Aktivitäten oder interaktiven H5P-Übungen, wo repetitive Übungen ${ }^{1}$ mit automatisiertem Feedback und die Anpassung der Lerngeschwindigkeit an individuelle Bedürfnisse möglich sind. H5P ist ein externes Open-Source Autor*innentool, mit dem sich sehr einfach solche interaktiven Übungen erstellen lassen, die aber direkt in Moodle integriert werden können und in allen Webbrowsern ohne $\mathrm{Zu}$ satzsoftware funktionieren. Bei Moodle- und H5PAktivitäten arbeiten Schüler*innen kaum noch mit Dateien, sondern lernen direkt anhand der interaktiven Übungen.

selbstgesteuert wiederholbare Übungen 
Einen höheren Mehrwert im Sinne einer Modifcation oder Redefinition würde der Einsatz von Technologie dann bringen, wenn er einen Einfluss auf die Pädagogik und Didaktik hat, was sich in modifizierten oder neu erdachten Aufgaben manifestieren würde. Dann würde es auch zu dem von Bubb und Jones (2020) beschriebenen positiven Effekt kommen.

Beispiele für solche modifizierten und neu-definierten Aufgaben wären:

1. Adaptive, individualisierte Lernpfade,

2. kooperative Datensammlung mit Interpretation,

3. Verschränkung von kooperativen Aufgaben mit spielbasierten Lernsequenzen, wie bspw. mit Glossaren und Moodle Games oder

4. Motivationsförderung durch Gamification-Elemente mit LevelUp! oder Stash.

Diese Beispiele lassen sich sehr gut mit den in Moodle vorhandenen Werkzeugen abdecken und bieten daher das Potential, einen hohen Mehrwert für das Lernen durch den Einsatz von Technologie zu erreichen.

Für die Umsetzung adaptiver, individualisierter Lernpfade können in Moodle mittels sog. „Voraussetzungen“"verschiedene Regeln hinterlegt werden, über welche Lernpfade vordefiniert werden können. Gekoppelt mit automatisiertem Feedback können Schüler*innen daher weitgehend autonom die Lernangebote in Anspruch nehmen; die Lehrpersonen behalten in ihrer Rolle als Lernbegleiter*in über die Moodle-Berichte den Lernfortschritt im Auge und können auf individueller Basis unterstützen.

Kooperative Datensammlungen können beispielsweise Rechercheergebnisse in Form von Wikis oder Datenbanken darstellen, die durch die Lernenden zusammengetragen und in einem gemeinsamen Reflexionsprozess interpretiert werden.

Viele Moodle-Aktivitäten lassen sich miteinander kombinieren, wie beispielsweise Glossare und Moodle-Games. Lässt man Lernende ein gemeinsames Glossar erstellen, so müssen sie sich recht intensiv mit einem Thema auseinandersetzen, um bestimmte Begriffe so erklären zu können, dass es die anderen Lernenden verstehen. Im Anschluss an die Erstellung des Glossars können die Begriffe dann zum Beispiel in Form von Kreuzworträtseln, Suchworträtseln uvm. zur Übung freigegeben werden. Dabei unterscheidet sich jeder Durchlauf vom Vorherigen, und jede*r Lernende erhält eigene Übungen. Als Lernbegleiter*in liegt also der Fokus in der Vorbereitung des Lernpfades, der passenden Formulierung der Aufgabenstellung und der Begleitung des Lernfortschritts durch Feedback und eine Qualitätskontrolle der Eingaben seitens der Lernenden.

Die angesprochenen Gamification-Elemente stellen im Gegensatz zu den Games selbst kein Spiel dar.
Allerdings werden bei Gamification einzelne Elemente aus Spielen entnommen und auf nicht-spielerische Kontexte angewandt, also bspw. bereits sehr rudimentär eine Highscore-Liste nach einem Quiz. Mit LevelUp! erhalten die Lernenden Erfahrungspunkte und können Level hochsteigen, bei Stash hingegen kann man virtuelle Gegenstände verstecken, die die Lernenden dann sammeln und tauschen können.

Diese Beispiele zeigen, wie man auch herkömmliche Aufgaben durch eine Transformation von Pädagogik und Didaktik anreichern kann. Allerdings erfordert die Umsetzung solcher Verbesserungen von den Lehrer*innen viel Know-how, und nicht zuletzt muss man fast alle bisher genutzten Übungsbeispiele adaptieren.

Bezogen auf die beiden Prämissen, dass viele Lehrer*innen durch die Schulschließungen erst mit digitalem Unterricht begonnen haben und zugleich bei den SAMR-Level Ersetzung (Substitution) bzw. Verbesserung (Augmentation) geblieben sind, da die Lehrpersonen hauptsächlich Videokonferenzen und Dokumentenaustausch (bspw. Arbeitsblätter) eingesetzt haben, müsste sich das an der Anzahl der genutzten Moodle-Aktivitäten und hochgeladenen Dateien zeigen. Daher wird im Weiteren untersucht, wie sich die Anzahl der Moodle-Aktivitäten und der hochgeladenen Dateien durch die Schulschließungen verändert hat.

\section{Die Verbreitung und Entwicklung der Lernplattform eduvidual.at}

Die Lernplattform eduvidual.at wird vom österreichischen Bildungsministerium für Schulen zentral bereitgestellt. Bis Ende 2021 werden alle Schulen aus dem Vorgängerprojekt lernplattform.schule.at (ebenfalls eine Plattform mit Moodle-Technologie) in die neue Lernplattform migrieren. Bei lernplattform.schule.at werden daher keine neuen Schulen mehr angelegt, neue Schulen müssen sich bei eduvidual.at registrieren. Beide Moodle-Plattformen zusammen werden in diesem Artikel als „Bundes-Moodle“ bezeichnet. Neben der Nutzung von Moodle wird vom österreichischen Bildungsministerium noch die Lernplattform lms.at angeboten. In den letzten Jahren wurde zusätzlich vom Ministerium und von den Bildungsdirektionen verschiedener Bundesländer auch die Nutzung von Microsoft Office 365 und Google GSuite beworben. Es wurde ein eigenes Serviceportal eingerichtet, um Schulen eine Entscheidungsgrundlage zu bieten, bei denen die genannten vier Alternativen vorgestellt und verglichen werden (Bundesministerium für Bildung, Wissenschaft und Forschung 2020).

Vor einem Jahr, also nach den ersten Schulschließungen von März-April 2020, wurde bereits 
über die aktuelle Verbreitung des österreichischen Bundes-Moodle berichtet (Schrenk 2020: $52 \mathrm{f}$.). In der Zwischenzeit gab es in Österreich zwei weitere Lockdowns, die sich auf die Anzahl an Schulen bei eduvidual.at ausgewirkt haben. In den nachfolgenden Tabellen und Diagrammen sind eduvidual.at, lernplattform.schule.at und Bundes-Moodle getrennt ausgewiesen, weil manche Schulen die beiden Plattformen eduvidual.at und lernplattform.schule.at parallel verwenden können. Die Spalte „Anteil“ bezieht sich daher auf die Anzahl der Schulen im Bundes-Moodle bezogen auf die Gesamtanzahl an Schulen im jeweiligen Bundesland. Die Zahlen in Klammern geben die Veränderung gegenüber dem Bericht von 2020 an (Schrenk 2020: 52 f.).

Ein besonders starkes Wachstum war in Vorarlberg $(+4,4 \%)$, Wien $(+3,9 \%)$, Tirol $(+3,0 \%)$ und der Steiermark $(+2,8 \%)$ zu verzeichnen. Gemessen an der Anzahl der Schulen ist eduvidual.at im letzten Jahr von 646 auf 948 um 46,7\% gewachsen. Das österreichische Bundes-Moodle versorgt somit fast 20\% aller Schulen. Die Änderung bei lernplattform.schule. at betreffen Moodle-Instanzen, die zuvor keinem oder einem falschen Bundesland zugeordnet waren. $\mathrm{Zu}$ sätzlich werden von einzelnen Bundesländern, wie bspw. Tirol und Oberösterreich, aber auch von manchen Schulen selbst eigene Moodle-Lernplattformen betrieben. Die Zahlen dieser Lernplattformen sind allerdings nicht bekannt und müssten bei einer Gesamtbetrachtung der österreichischen LernplattformSzene mitberücksichtigt werden.

Nach Schultypen aufgeschlüsselt verzeichnete $e d u$ vidual.at einen starken Zuwachs im Bereich der Gymnasien $(+5,4 \%)$ und Handelsakademien $(+4,2 \%)$. Allerdings sind auch viele Berufsschulen $(+3,8 \%)$ und Mittelschulen (+ 3,8\%) bei eduvidual.at eingestiegen. Bemerkenswert ist, dass eduvidual.at mittlerweile bei mehr als $70 \%$ aller österreichischen Gymnasien im Einsatz ist.

Der Zuwachs von 302 Schulen bei eduvidual.at und 146 beim Bundes-Moodle lässt den Schluss zu, dass 156 Schulen von lernplattform.schule.at zu eduvidual.at übersiedelt und 146 Schulen neu zu Moodle (eduvidual.at) hinzugekommen sind. 278 Schulen von lernplattform.schule.at werden noch im Jahr 2021 in das gemeinsame eduvidual.at-System migrieren.
Tab. 1: Bundes-Moodle nach Region (Stand 19.03.2021)

\begin{tabular}{|c|c|c|c|c|c|}
\hline Bundesländer & Anteil & $\begin{array}{c}\text { Schu- } \\
\text { len } \\
\text { gesamt }\end{array}$ & $\begin{array}{l}\text { Bundes- } \\
\text { Moodle }\end{array}$ & $\begin{array}{l}\text { eduvidu- } \\
\text { al.at }\end{array}$ & $\begin{array}{l}\text { lernplatt- } \\
\text { form. } \\
\text { schule.at }\end{array}$ \\
\hline Burgenland & $3,5 \%(+0,7)$ & 286 & $10(+2)$ & $6 \quad(+2)$ & 6 \\
\hline Kärnten & $12,1 \%(+1,2)$ & 497 & $60(+6)$ & $43(+11)$ & 43 \\
\hline Niederösterreich & $11,2 \%(+2,1)$ & 1263 & $141(+26)$ & $106(+43)$ & 76 \\
\hline Oberösterreich & $29,6 \%(+1,7)$ & 1109 & $328(+118)$ & $229(+88)$ & 270 \\
\hline Salzburg & $39,3 \%(+2,2)$ & 410 & $161(+9)$ & $149(+13)$ & 46 \\
\hline Steiermark & $20,8 \%(+2,8)$ & 934 & $194(+26)$ & $151(+50)$ & $118(+1)$ \\
\hline Tirol & $9,1 \%(+3,0)$ & 694 & $63(+21)$ & $37(+23)$ & $35(+1)$ \\
\hline Vorarlberg & $10,6 \%(+4,4)$ & 292 & $31(+13)$ & $28(+13)$ & 3 \\
\hline Wien & $33,9 \%(+3,9)$ & 703 & $238(+25)$ & $199(+61)$ & $135(+1)$ \\
\hline Summe & & 6188 & $1226(+146)$ & $948(+302)$ & $732(+2)$ \\
\hline
\end{tabular}

Tab. 2: Bundes-Moodle nach Schultyp (Stand 19.03.2021)

\begin{tabular}{|c|c|c|c|c|c|}
\hline Schul & Anteil & $\begin{array}{l}\text { Schu- } \\
\text { len } \\
\text { gesamt }\end{array}$ & $\begin{array}{l}\text { Bundes- } \\
\text { Moodle }\end{array}$ & $\begin{array}{l}\text { eduvidu- } \\
\text { al.at }\end{array}$ & $\begin{array}{l}\text { lernplatt- } \\
\text { form. } \\
\text { schule.at }\end{array}$ \\
\hline 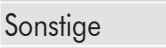 &, $2 \%(+1,8)$ & 493 & $65(+9)$ & $49(+20)$ & 42 \\
\hline VS & $4,5 \%(+1,5)$ & 3155 & $143(+49)$ & $123(+53)$ & 31 \\
\hline HS/NMS/MS & $38,3 \%(+3,8)$ & 1120 & $429(+52)$ & $345(+91)$ & 235 \\
\hline Sonderschule & $16,6 \%(+1,5)$ & 265 & $44(+4)$ & $41 \quad(+7)$ & $91+$ \\
\hline TS & $26,8 \%(+1,2)$ & 179 & $48 \quad(+2)$ & $38 \quad(+8)$ & $221+$ \\
\hline BS & $6,8 \%(+3,8)$ & 153 & $41 \quad(+6)$ & $32(+10)$ & 28 \\
\hline Jymn & $0,9 \%(+5,4)$ & 371 & $263(+21)$ & $186(+79)$ & 209 \\
\hline HTL & $27,3 \%(+2,0)$ & 150 & $41 \quad(+3)$ & $30(+8)$ & 33 \\
\hline HAK & $52,9 \%(+4,2)$ & 119 & $63(+5)$ & $40(+13)$ & 52 \\
\hline HUM & $48,6 \%(+2,2)$ & 183 & $89 \quad(+4)$ & $64(+15)$ & 69 \\
\hline Summe & & 6188 & $1226(+146)$ & $948(+302)$ & 732 \\
\hline
\end{tabular}

\section{Die Verbreitung und Entwicklung verschiedener Moodle-Aktivitäten}

Insgesamt bietet eduvidual.at den Lehrer*innen eine Auswahl aus 58 Aktivitäten (Foren, Aufgaben, Games, Quiz, ...) und neun Ressourcen (Dateien, URLs, ...) an. Mit diesen Aktivitäten und Ressourcen lassen sich Szenarien auf allen Ebenen des SAMR-Modells umsetzen.

So werden im Fall der Lernplattform eduvidual.at die Videokonferenzlösungen BigBlueButton und MS Teams direkt unterstützt und man kann Dateien zum Download anbieten sowie Abgaben von Schüler*innen mittels Moodle-Aktivitäten wie der „Aufgabe“ wieder einsammeln (Ersetzung/ Substitution).

Andere Moodle-Aktivitäten bieten die Möglichkeit einen höheren Mehrwert für das Lernen zu generieren, also die SAMR-Level Modifikation (Modification) und Neudefinition (Redefinition) zu erreichen. Beispiele dafür wären die bereits beschriebene didaktische Verschränkung von Aktivitäten, wie ein kooperativ erstelltes Glossar mit daran anschließenden Moodle-Games zur Festigung der Lerninhalte oder ein kooperativ erstellter Fragenpool mit wechselseitiger Feedbackmöglichkeit. Bei beiden genannten Beispielen liefert die Lehrperson lediglich das didaktische Framework. Die Lehrinhalte werden durch die Lernenden selbst eingebracht und von der Lehrperson 


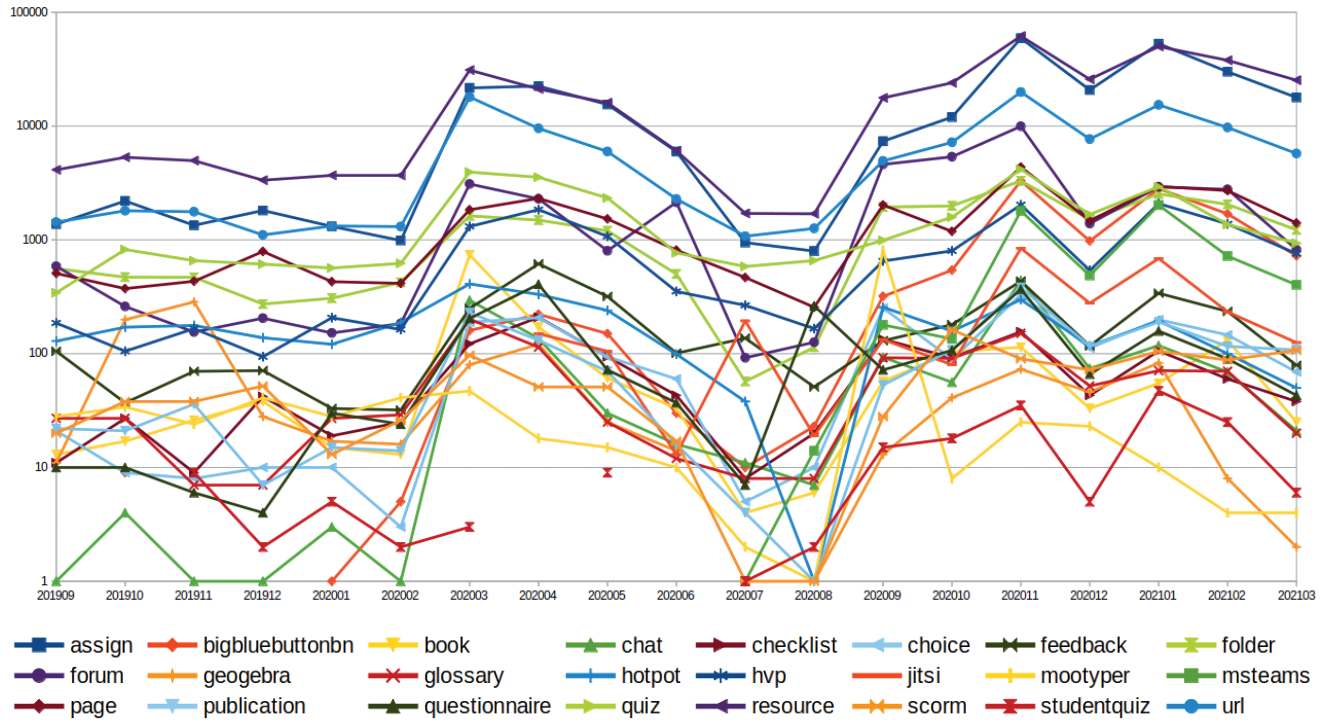

Abb. 2: Anzahl an erstellten Aktivitäten nach Typ (eigene Darstellung) ggfs. qualitätsgesichert. Dieser sozialkonstruktivistische Ansatz von Pädagogik stellt sogar das Leitprinzip von Moodle dar (Hillenbrand \& Schlindwein 2017)!

Im Weiteren wird analysiert, wieviele Aktivitäten und Ressourcen in jedem Monat zwischen September 2019 und Februar 2021 von Lehrpersonen erstellt wurden. Abb. 2 zeigt logarithmisch die Entwicklung jener Aktivitäten und Ressourcen, von denen im gesamten Betrachtungszeitraum zumindest 1000 erstellt wurden.

Die Abb. 2 zeigt deutlich, wie sich die SchulschlieBungen im März/April 2020, November 2020 und Jänner 2021 ausgewirkt haben. Die hauptsächlich genutzten Aktivitäten und Ressourcen waren Dateien (resource), Aufgaben (assign), Hyperlinks (url) und zumindest beim zweiten Lockdown auch Foren (forum).

Ein Detailvergleich zwischen November 2019 und 2020 zeigt, dass 64-mal soviele Foren, 44-mal so- viele Aufgaben, 41-mal soviele Abstimmungen und 22-mal soviele Glossare erstellt wurden. Auch auf die Mischung zwischen herkömmlichen und neuartigen Aufgaben hat diese Änderung einen Einfluss. Während im November 2019 auf ein Glossar noch 192 Aufgaben erstellt wurden, waren es im November 2020 pro Glossar sogar 392 Aufgaben. Bei Moodle Games hat sich dieses Verhältnis von 1:134 sogar auf 1:758 vergrößert.

Auch bei der Anzahl der Dateien lässt sich ein klarer Trend ablesen: Abb. 3 zeigt die Anzahl der Dateien pro Anwendungsgebiet und illustriert auf einer logarithmischen Skala, dass PDF-Feedbacks vor den Schulschließungen mehr oder weniger irrelevant waren, aber seitdem deutlich an Popularität dazugewonnen haben.

Während beim ersten Lockdown zwischen März und April 2020 diese Funktion noch moderat genutzt wurde, war ein starker Anstieg im Mai 2020 zu ver-

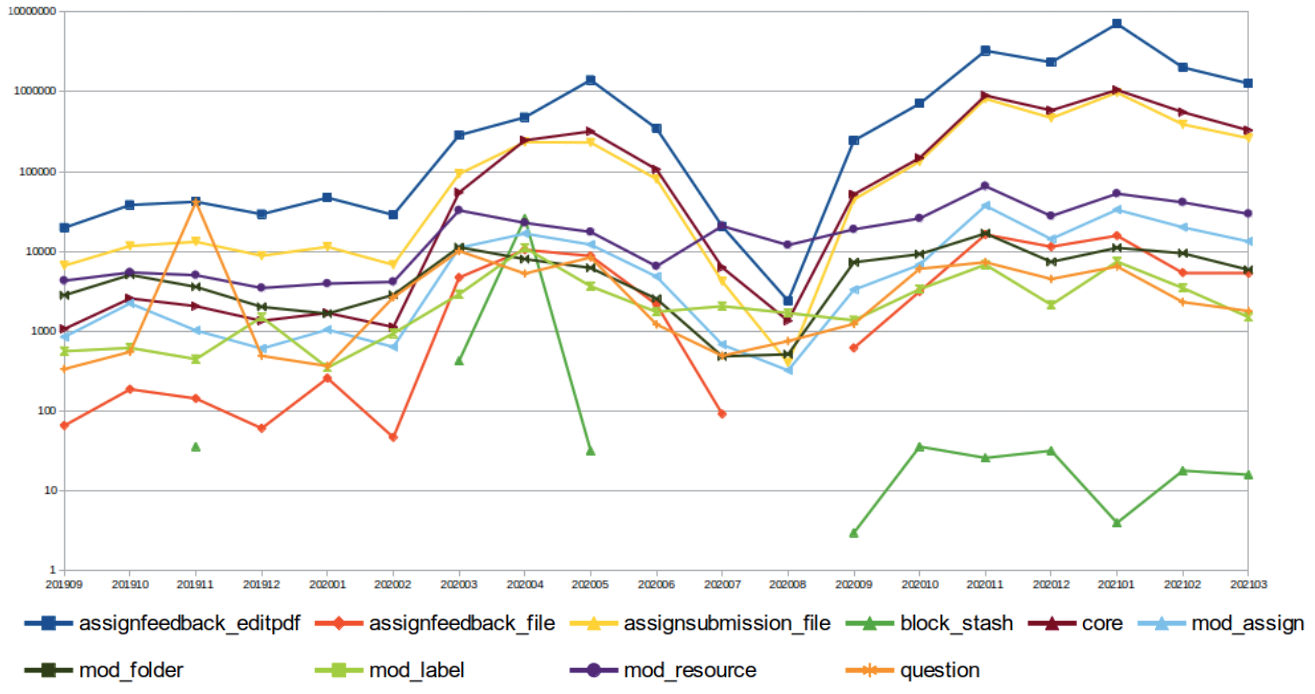

Abb. 3: Anzahl an hochgeladenen Dateien nach Aktivität (eigene Darstellung) 
zeichnen, obwohl hier alle Schulen geöffnet waren. Im Mai 2020 wurden etwa 1.400.000 PDF-Feedbacks durchgeführt. Im November 2020 waren es bereits 3.100.000 und im Jänner 2021 sogar über 7.000.000. Nachdem die Schulen ab Februar wieder offen waren, ist diese Anzahl drastisch zurückgegangen. Insofern kann der Begriff der „PDF-Schleuder“ für diese Art der Anwendung durchaus zur Anwendung kommen, auch wenn sie in diesem Fall zumindest schon in Form von Rückmeldungen der Lehrperson zu den Lernenden genutzt wurde.

Als Kritik an dieser Analyse muss anerkannt werden, dass die Art des Werkzeugs (bzw. der MoodleAktivität) nicht zwangsläufig einen Rückschluss auf die Qualität des Lernszenarios zulässt. Die Art der Aktivität allein stellt keinen Nachweis für bestimmte pädagogische Methoden dar und auch das Aufgabenmodul kann für modifizierte oder neudefinierte Aufgaben herangezogen werden. Methoden wie das kooperative, offene Lernen (COOL) funktionieren sogar ganz ohne digitale Technologie und auch diese könnten mit gewöhnlichen Moodle-Aufgaben umgesetzt werden, bei denen die Lernenden Dokumente hochladen. Der Arbeitsaufwand ist für Lehrpersonen dann allerdings ungleich höher. Gerade hier könnte die digitale Technologie durch Automatismen und Berichte die Lehrpersonen massiv unterstützen.

Eine qualitative Erhebung von Oberrauch, Jekel und Breitfuss-Horner (in Druck) bestätigt das Ergebnis dieser Analyse. Die befragten Lehrpersonen berichten, dass in ihrem Umfeld trotz erweiterter digitaler Möglichkeiten hauptsächlich Aufgaben im Bereich der Reproduktion aufgegeben werden und nicht solche aus den Bereichen der Anwendung oder Reflexion. Als Gründe dafür wird genannt, dass solche Aufgaben, die höhere Anforderungsbereiche betreffen, als kaum oder nur „mühsam“ umzusetzen angesehen werden. In den von Lehrpersonen genannten "To do's" wird unter anderem explizit die Möglichkeit zu kooperativen und kollaborativen Aufgaben angesprochen, die auch nach der Moodle-Philosophie so zentral wären (Hillenbrand \& Schlindwein 2017).

Jekel, Oberrauch und Breitfuss-Horner (2020: 64) identifizierten, dass „vorgefertigte Beispiele, bei denen nur mehr die Diagramme, Statistiken oder Artikel selbst aktualisiert werden müssen, wünschenswert und für die Lehrpersonen als Hinführung hilfreich [wären]“. Für diese Zwecke wurde beispielsweise in eduvidual.at ein öffentlicher Ressourcenkatalog eingerichtet, in dem Lehrer*innen offene Bildungsressourcen (OER) publizieren können (eduvidual.at o. J.). Die OER-Initiative des National Competence Center eEducation Austria (o. J.) namens e-Tapas wird in edu- vidual.at abgewickelt, da die Autor*innen diese Ressourcen direkt in eduvidual.at erstellen und freigeben können, und auch das Peer Review dieser Ressourcen in eduvidual.at durchgeführt wird. Somit stehen eine große Zahl an qualitätsgesicherten Kursen und Unterrichtsressourcen zur Verfügung. Es wäre daher wünschenswert, wenn solche Angebote in Zukunft auch vermehrt eingesetzt würden.

\section{Fazit}

Die eingangs erwähnten Prämissen scheinen bestätigt: Lehrer*innen, die erstmalig mit digitalen Technologien arbeiten, tendieren dazu, herkömmliche Lehrmuster und -materialien 1:1 aufs Digitale zu übertragen. Diese Annahme wurde durch qualitativ erhobene Erkenntnisse von anderen Autor*innen bestätigt (Oberrauch, Jekel \& Breitfuss-Horner in Druck). Darüber hinaus legt auch der enorme Zuwachs bei Dateien, URLs und Aufgaben bei einem im Vergleich dazu geringen Zuwachs an Glossaren, Games und anderen fortgeschrittenen Aktivitäten einen solchen Schluss nahe. Auf dieser Ebene des Einsatzes von Technologie (Substitution) entsteht aber nur ein geringer Mehrwert für das Lernen durch die Technologie.

Im Gegensatz zum Bericht der norwegischen Schulen wäre es demnach in Österreich nicht zu einer flächendeckenden Transformation der Pädagogik durch die Digitalisierung gekommen. Es scheint eher so, als wäre in Österreich der Unterricht genauso wie zuvor weitergelaufen, nur dass er für kurze Zeit auf digitale Kanäle übertragen wurde.

Zhao (2020: 30) beschreibt die COVID-19 Pandemie als Chance, um Pädagogik neu zu denken. $\mathrm{Zu}$ befürchten ist leider, dass diese Chance in Österreich nicht ergriffen wurde und wird.

Ein weiterer Einsatz von digitalen Technologien, insbesondere die Verwendung von Moodle-Aktivitäten und weiteren Möglichkeiten, wie adaptive Lernszenarien, Gamification etc., könnten einen positiven Einfluss auf Pädagogik, Methodik und Didaktik haben. Dazu ist es aber notwendig, dass Lehrer*innen nicht einfach in alte Muster zurückfallen, sondern, sobald die Pandemie unter Kontrolle ist, in der Erkenntnis, dass hier Verbesserungen möglich sind, an dem Thema interessiert bleiben und sich fortbilden.

Die zentrale Herausforderung bei der Digitalisierung ist die Transformation der Pädagogik. Ein gut überlegter Einsatz von Technologie, bei dem das SAMR-Modell ein nützliches Reflexionswerkzeug darstellt, kann als Triebfeder zur Verbesserung der Pädagogik dienen. Digitalisierung allein ist aber kein Garant für Verbesserung. 


\section{Literatur}

Bubb, S. \& M.-A. Jones (2020): Learning from the COVID-19 home-schooling experience: Listening to pupils, parents/carers and teachers. Improving Schools 23(3). S. 209-222. https://doi.org/10.1177/1365480220958797

Bundesministerium für Bildung, Wissenschaft und Forschung (2020): Serviceportal eEducation: Distance Learning Serviceportal. https://serviceportal.eeducation.at/ (18.04.2021)

eduvidual.at. (o. J.): Ressourcenkatalog. https://www. eduvidual.at/blocks/edupublisher/pages/search.php (13.04.2021)

NCoC e-Education Austria. (o. J.): eEducation: e-Tapas. https://eeducation.at/ressourcen/etapas (13.04.2021)

Hillenbrand, G. \& B. Schlindwein (2017). Moodle Pädagogik. https://docs.moodle.org/38/de/P\%C3\%A4dagogik (18.04.2021)

Jäger, P., A. Kieffer, A. Lorenz \& N. Nistor. (2014): Der Einfluss der didaktischen Gestaltung auf die Akzeptanz und Nutzung von moodle in der Hochschullehre. In Lernräume gestalten - Bildungskontexte vielfältig denken. Waxmann, Münster. S. 485-495.

Jekel, T., A. Oberrauch \& C. Breitfuss-Horner (2020): „Ich habe unbekannte Seiten und Talente meiner Schüler/innen entdeckt "Eine Delphi-Studie zum Ist-Stand und Entwicklungsstrategien zur fachspezifischen Fernlehre an österreichischen Sekundarschulen. In: GW-Unterricht 158. S. 57-67. https://doi.org/10.1553/gw-unterricht158s57
Madner, M. (2020): Schule - Warnung vor aufgehender Bildungsschere. Österreich Politik - Nachrichten -Wiener Zeitung Online. https://www.wienerzeitung.at/ nachrichten/politik/oesterreich/2058419-Warnung-voraufgehender-Bildungsschere.html (18.04.2021)

Oberrauch, A., T. Jekel \& C. Breitfuss-Horner (in Druck): Lehrer*innenperspektiven auf die Digitalisierung von (geographischer) Bildung. In: Pettig, F. \& I. Gryl: Geographische Bildung in digitalen Kulturen. Perspektiven für Forschung und Lehre. Springer, Heidelberg.

Ottacher, G., I. Aichhorn, M. Zak, S. Seyrling, H. Ebner, C. Friedl, M. El-Roumy, J. Moser, Y. Teufel, M. Strauch \& Ö. Çatı kkaş (2020): Bildungsgerechtigkeit in Zeiten von Corona - Was wir tun. Teach For Austria. https:// www.teachforaustria.at/story/bildungsgerechtigkeittrotz-corona/ (18.04.2021)

Puentedura, R. (2006): Transformation, technology, and education. http://hippasus.com/resources/tte/ (18.04.2021)

Puentedura, R. (2013): SAMR: Moving from Enhancement to Transformation. http://www.hippasus.com/ rrpweblog/archives/2013/05/29/SAMREnhancementToTransformation.pdf (18.04.2021)

Schrenk, R. (2020): Distance Learning mit Moodle - Aktuelles aus Österreichs Schulen. GW-Unterricht 158. S. 51-56. https://doi.org/10.1553/gw-unterricht158s51

Zhao, Y. (2020): COVID-19 as a catalyst for educational change. PROSPECTS 49(1-2). S. 29-33. https://doi. org/10.1007/s11125-020-09477-y 